\title{
Transboundary Watershed Pollution Control and Product Market Competition With Ecological Compensation and Emission Tax: A Dynamic Analysis
}

yongxi yi ( $\nabla$ yyx19999@126.com )

University of south china https://orcid.org/0000-0002-2479-4488

Caini Ding

University of South China

Chunyan Fu

University of South China

Yuqiong Li

University of South China

\section{Research Article}

Keywords: Pollution control, Ecological compensation, Market competition, River basin

Posted Date: October 28th, 2021

DOI: https://doi.org/10.21203/rs.3.rs-1020578/v1

License: (c) (1) This work is licensed under a Creative Commons Attribution 4.0 International License.

Read Full License 


\title{
Transboundary watershed pollution control and product market competition
} with ecological compensation and emission tax: A dynamic analysis

\author{
Yongxi Yi*; Caini Ding; Chunyan Fu; Yuqiong Li \\ School of Economics Management and Law, University of South China, China \\ *Corresponding Author: Yongxi Yi, email: yyx19999@126.com
}

Abstract: Product competition and pollution control are closely related to watershed environmental management, but existing literature rarely investigates them in an identical framework. Therefore, this paper develops a multiple differential game model to analyze product market competition and ecological compensation games between watershed regions based on the assumption that a region can choose four strategies to regulate its manufacturers while cooperating with other regions in the basin. Then, solve the model and obtain a simultaneous equilibrium between the governments and manufacturers for the first time. The results show that: the combination of emissions tax and ecological compensation results in the highest social welfare and water ecology for all regions in a basin. Furthermore, the ecological compensation rate independent of emissions tax policy and ecological compensation does not shift investment from downstream to upstream, but it can induce the upstream region to increase investment in management. In addition, if the governments impose an emissions tax, manufacturers' output in both regions decreases, and the upstream region is higher than in the downstream region.

Key words: Pollution control; Ecological compensation; Market competition; River basin

\section{Introduction}

Pollutants with the water bodies flowing from upstream to downstream in river basins bring countless transboundary pollution problems with dual externalities. As a result, they have caused significant challenges to river basins' environmental governance (Jiang et al., 2019). On the one hand, emission in an upstream generates adverse external effects to its downstream; conversely, pollution abatement can make the water environment in downstream regions cleaner, creating positive externalities (Yi et al., 2021). According to Pigou's pioneering work (1921), negative externalities incite polluters to release too many pollutants, while positive externalities make 
pollution reducers abate too few pollutants relative to the optimal level of society. So these two external influences reduce social welfare and worsen the water ecological environment (Yi et al., 2021).

Moreover, transboundary pollutions in river basins involve two or more regions, so unilateral efforts cannot solve these multilateral issues. They are incredibly dependent on close cooperation between the upstream and downstream of the river basin (Peng et al., 2014; Zeng et al., 2019). In practice, some cooperative mechanisms for transboundary pollution control have emerged. Among them, ecological compensation may be one of the most concerned governance mechanisms in China. For example, the Chinese government issued the "Opinions on Improving the Compensation Mechanism for Ecological Protection” in May 2016. The “Opinions” clearly require that by 2020, achieve full coverage of ecological protection and compensation in critical areas such as forests, grasslands, wetlands, deserts, oceans, water flows, cultivated land, prohibited development areas, and critical ecological function areas. Following the requirements of the central government, local governments in some river basins in China have taken the lead in implementing ecological compensation practices in the Xin'an River, Jiuzhou River, Tingjiang-Hanjiang River, Dongjiang River, Luanhe River, and Weihe River Basin. Other basins that have not yet implemented ecological compensation are actively preparing.

Although ecological compensation has been carried out in many river basins, achieving the expected results is another question. The key to its success lies in the design of the mechanism (Jiang et al., 2019). The local governments of the river basin have to carry out pollution reduction and ecological compensation because polluters discharge pollution. Therefore, the design of the ecological compensation mechanism should consider the possible response of the significant polluters, i.e., manufacturers. In other words, it should base on weighing the behavior of the government and the manufacturers. However, the relevant research so far has not considered the manufacturer's response to ecological compensation, nor has it considered the possible impact of changes in the manufacturer's pollutant discharge behavior on the optimal ecological compensation mechanism. In order to bridge this gap, this paper incorporates the behavior of manufacturers into the analysis framework of watershed ecological compensation. Moreover, as shown in the Fig.1, we build a triple game model that includes (1) a game between regions. (2) a game between regional governments and their manufacturers. (3) a game between manufacturers 

the first time.

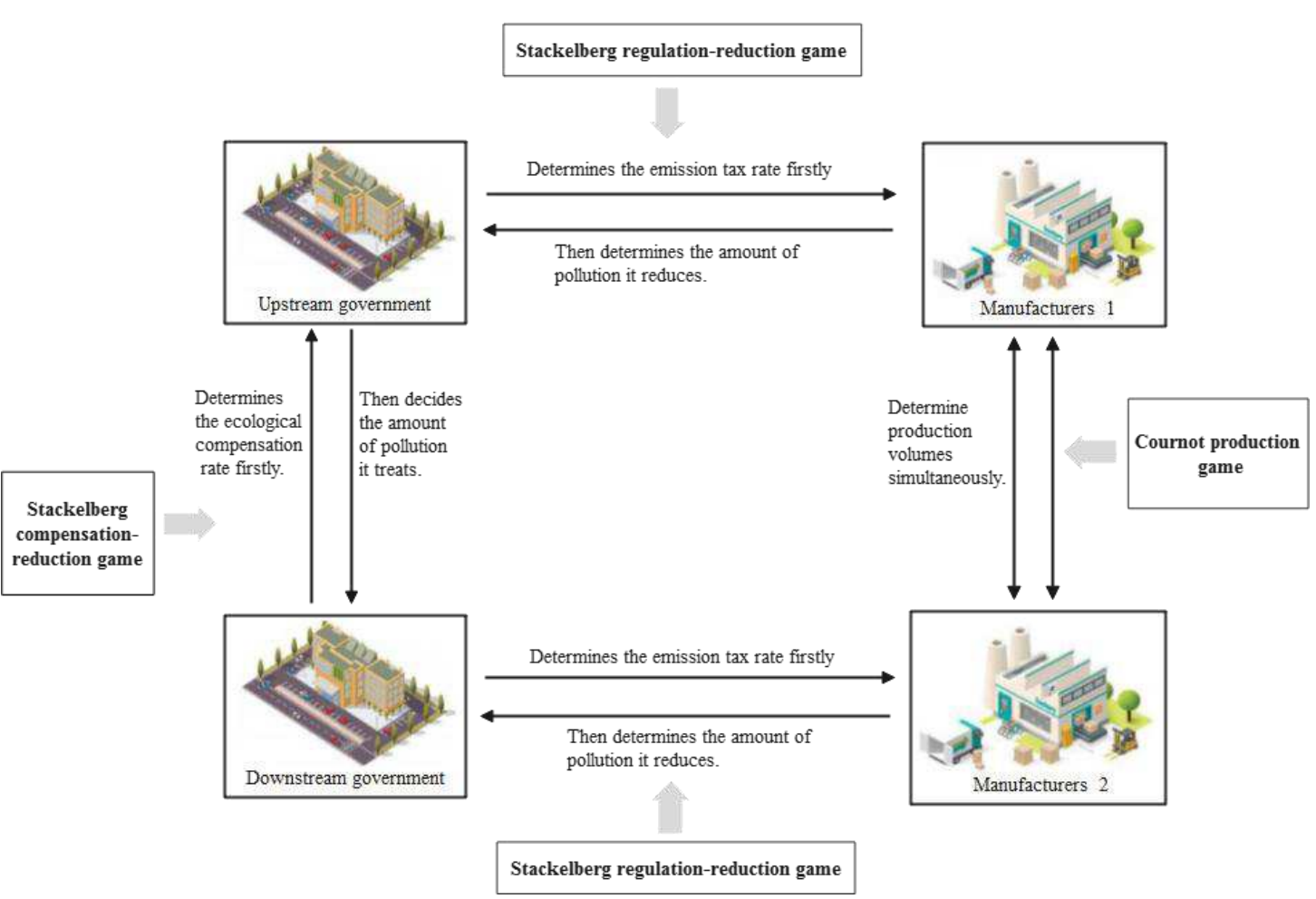

in various regions. Furthermore, through game analysis, we obtain a simultaneous equilibrium of the regional governments and manufacturers in the ecological compensation in a river basin for

Fig.1 Game structure diagram of the study

\section{Literature review}

The problem of transboundary pollution appears and becomes more and more severe with the development of industrialization. In this regard, the academic community has been studying it since the early 1990s. For example, Long (1992) investigates transboundary pollution control between two symmetric countries using the differential game method and finds that the cooperative strategy results in a cleaner environment than the open-loop Nash equilibrium strategy. Along the path of Long (1992), Ploeg et al. (1992), Dockner et al. (1993), and Yeung (2007) also find that cooperation in various regions involving transboundary pollution can achieve better results than non-cooperation. Carraro and Siniscalco (1993) and Breton et al. (2010) investigate the stability of cooperation in transboundary pollution control on the condition that the 
participants obtain a higher present value of welfare level at each point in time when cooperating than when not cooperating. In addition, Peng et al. (2014) and Zeng et al. (2019) find through game analysis that unilateral actions can not solve transboundary pollution and further emphasize the critical role of cooperation in transboundary pollution control. Huang et al. (2016) use a differential game model to investigate the cooperation strategies of transboundary pollution control in two adjacent regions. The authors show that if they choose to cooperate, each region can obtain higher payments at any point in time than if they did not cooperate. Li et al. (2019) analyze the transboundary pollution control decisions of the local governments in a basin under the emission rights trading and find that the cooperation mechanism leads to increased investment in pollution reduction in the upstream region. From the perspective of global pollution control, Boucekkine et al. (2021) studied the temporal and spatial characteristics of transboundary pollution and the optimal pollution control investment and emission strategies of various countries. Using the successful air transboundary pollution control cases between Hong Kong and Guangdong Province in China, Jiao et al. (2021) analyze the key factors leading to successful cooperation in pollution control. De Frutos et al. (2021) use parabolic partial differential equations to define the dynamic characteristics of pollutant stock and investigate the dynamic game strategies involving participants in multi-regional transboundary pollution. The study shows that the optimal strategy of the participants is affected by geographical factors.

The research mentioned above fully proves that the effective control of transboundary pollution requires the active cooperation of all parties involved. Therefore, how to implement cooperation is another critical issue. In recent years, with the development of practice and the deepening of research, ecological compensation as a cooperative governance mechanism has become a research hotspot. Muradian and Corbera (2010), Cranford and Mourato (2011), and Kemkes et al. (2010) analyze the meaning and rationality of ecological compensation and put forward some proposals for implementing ecological compensation from different perspectives. Lu et al. (2020) raise a method for determining the ecological compensation standard of a river basin based on calculating the economic loss of transboundary pollution. Gao (2019) uses game analysis to study the construction of an ecological compensation mechanism for the East Route of South-to-North Water Transfer Project in China. Jiang et al. (2019) consider the effect of learning by doing in pollution-reducing and analyze the ecological compensation standard in a river basin. 
At the same time, they show that ecological compensation can effectively encourage upstream regions to reduce pollution. Yi et al. (2020a) find that ecological compensation in a river basin can result in a Pareto improvement result. Using the evolutionary game method, Shen et al. (2021) analyze the construction of the ecological compensation mechanism in the Taihu Lake Basin. Yi et al. (2021) analyze local governments' pollution reduction and ecological compensation strategies in ecological compensation in a river basin with multiple upstream and downstream regions through a game model.

In addition to the ecological compensation strategy, another strategy studied in this paper is to encourage manufacturers to reduce emissions through emission taxes. This environmental policy has been widely used and extensively studied since Pigou (1932). Early research on emission tax, such as Buchanan (1969) and Barnett (1980), does not support the view that it raise social welfare. However, subsequent studies, such as Xepapadeas (1992), Feichtinger et al. (2016), and Lambertini et al. (2017), tend to recognize its positive effects. Recent related research mainly focuses on the optimal mechanism design. For example, Martín-Herrána and Rubio (2018) find that the optimal emission tax rate depends on the difference between pollution stocks' social and private shadow prices. Chen et al. (2018) apply evolutionary game theory to study the behavior of government and manufacturers and the design of policy mechanisms under carbon emission tax and subsidy policies. Wei et al. (2019) analyze the green innovation behavior of manufacturers under the pollution tax and propose an optimal emission tax for regulation oligopoly manufacturers. Yi et al. (2020b) believe that the combination of pollution tax and green innovation subsidies can improve social welfare.

In summary, the recent related research literature has shed light on ecological compensation in the river basin. However, a problem in these studies is that they only consider the government's behavior and rarely involve the role of manufacturers in ecological compensation and the impact of their behavior on the mechanism of ecological compensation. Therefore, this paper incorporates the regional governments and manufacturers' optimization issues into an analysis framework and constructs a game analysis model to study the internal regulation and external cooperation through ecological compensation in environmental governance for local governments in river basins.

The remainder of the article is as follows. First, the model grows up in Section 3. Then, Section 4 investigates the optimal behavior of manufacturers under four different policies and the 
optimal strategy of the governments and compares the welfare impact of four different policies.

139 Finally, Section 5 summarizes the paper.

\section{The model}

\subsection{Problem description}

Consider a watershed consisting of an upstream region and a downstream region where pollution crosses regional boundaries, and there is no administrative subordination between the upstream and downstream regions. In order to make the water bodies from the upstream cleaner, the downstream region is willing to compensate for a certain proportion of the pollution reduction investment in the upstream region. This kind of compensation is called river basin ecological compensation in this article. Like the practice in Xin'an River and Jiuzhou River basins in China, the downstream region carries out its pollution reductions and compensates for the upstream region simultaneously.

In practice, manufacturers are the leading force in pollution reduction. Therefore, the strategy of watershed ecological compensation inevitably needs to consider the behavior of manufacturers. Assume that all regions in the basin are in the same market, and the profit-maximizing manufacturers compete for the number of products. At the same time, each local government imposes a pollution tax on its manufacturers to encourage pollution reduction.

In order to obtain the full policy implications of the model analysis, this paper assumes that the regions in the river basin can freely choose to impose emission taxes on its manufacturers and freely choose to implement ecological compensation strategies. Therefore, there are following four combinations of strategies for each region: (no tax; no compensation); (no taxation; compensation); (tax; no compensation) and (taxation; compensation). Moreover, we also assume that the policies chosen by the two regions are the same. After all, if one region chooses to tax its manufacturers and the other does not, the manufacturers taxed will be at an extreme disadvantage. In addition, ecological compensation involves two or more regions, and only one region cannot make ecological compensation.

\subsection{Fundamental assumption}

In order to describe the model more clearly and conveniently, we first summarized the 
Table 1 Parameters and variables symbol descriptions

\begin{tabular}{|l|l|c|l|}
\hline \multicolumn{2}{|c|}{ Parameters } & & \multicolumn{1}{|c|}{ Variables } \\
\hline$a$ & Potential market size & $q_{i}(t)$ & Output of the Manufacturer $i$ at time $t$ \\
\hline$b$ & $\begin{array}{l}\text { Sensitivity of the price to the quantity of } \\
\text { the product }\end{array}$ & $p(t)$ & Price of the product at time $t$ \\
\hline$\phi$ & $\begin{array}{l}\text { Pollution transfer coefficient from an } \\
\text { upstream region to its downstream region }\end{array}$ & $k_{i}(t)$ & $\begin{array}{l}\text { Pollution reduce by the Manufacturer } \\
i \text { at time } t\end{array}$ \\
\hline$\eta$ & $\begin{array}{l}\text { Natural decay rate of water pollutants } \\
c\end{array}$ & $\begin{array}{l}\text { Marginal cost of the product } \\
\tau_{i}(t)\end{array}$ & Emission tax rate on Manufacturer $i$ \\
\hline$\varepsilon$ & marginal damage rate of pollution stock & $\delta$ & $\begin{array}{l}\text { Compensation rate of downstream the } \\
\text { region for its upstream region }\end{array}$ \\
\hline$\rho(0)$ & $\begin{array}{l}\text { Initial stock of water pollutants of } \\
\text { Region } i\end{array}$ & $s_{i}(t)$ & Pollution stock of Region $i$ at time $t$ \\
\hline & Risk-free rate & $u_{i}(t)$ & Pollution reduce of region $i$ at time $t$ \\
\hline
\end{tabular}

Let us start the problem description with a simple but widely used assumption, such as Li et al. (2019) and Yi et al. (2020), that a watershed consists of an upstream and a downstream region, denoted by $i, i=1,2$, where 1 refers to the upstream region, and 2 refers to the downstream region. The two regions may be within the same country, such as Anhui Province and Zhejiang Province, situated in the Xin'an River basin in China, or two neighboring countries. In addition, we assume that each region has one manufacturer that produces and sells a homogeneous product within the two regions, and they face a joint inverse demand function: 


$$
p(t)=a-b\left(q_{1}(t)+q_{2}(t)\right)
$$

where $p(t)$ is the product price at time $t, a>0$ stands for the potential market size, $q_{i}(t), i=1,2$ denotes the output of the Manufacturer $i$, and $b>0$ reflects the sensitivity of the price to the quantity of the product. Further, we assume that these two manufacturers compete in the Cournot marker to determine their optimal output $q_{i}^{*}(t)$ with the same marginal $\operatorname{cost} c$.

Every manufacturer inevitably needs to emit water pollutants during the production process. Consistent with Martín-Herrán et al. (2018) and Yi et al. (2020b), we assume that in the initial state, the amount of pollutants emitted by both manufacturer for each unit of product is 1 for simplicity, and it can be reduced by abatement investment $k_{i}(t)$ with $\cos t k_{i}^{2}(t) / 2$. This cost function has been widely used in the relevant literature such as Feichtinger et al. (2016) and Wei et al. (2019). Therefore, the instantaneous water pollutant discharged by Manufacturer $i$ equals to $q_{i}(t)-k_{i}(t)$. Given the pollution situation, suppose the governments in both regions are free choose to use emission taxes to encourage manufacturers to reduce emissions. If the emission taxes $\tau_{i}>0, i=1,2$, it means that the Region $i$ has applied the emission tax policy; $\tau_{i}=0$ indicates that the Region $i$ has given up using this policy.

Now, we can describe the instantaneous profits $\pi_{i}(t)$ of Manufacturer $i$ as follows considering all its benefits and costs mentioned above:

$$
\max _{q_{i}, k_{i}} \pi_{i}(t)=(p(t)-c) q_{i}(t)-\frac{k_{i}^{2}(t)}{2}-\tau_{i}(t)\left(q_{i}(t)-k_{i}(t)\right)
$$

While manufacturers compete for the number of products, the two regions can cooperate in pollution control employing ecological compensation. That is, in order to incentivize the upstream region to make pollution reductions to enjoy a cleaner water ecosystem, the downstream region is willing to compensate the upstream region for its treatment investments, with a compensation rate $\delta$. If $\delta>0$, it means that the two regions have implemented ecological compensation policies; $\delta=0$ indicates that the policy has not been carried out by the two regions. Concerning the research of Yi et al. (2021), we assume that the ecological compensation game is a Stackelberg game, in which the downstream region is the leader and determines the compensation rate. In 
contrast, the upstream region is the follower and makes reduction investment decisions at a given compensation rate. At the same time, the downstream region can also invest in pollution reduction. Assume that the amount of reduction is $u_{i}(t), i=1,2$, with the convex and increasing cost $u_{i}^{2}(t) / 2$. Such compensation and reduction model is standard in practices in China, such as the Xin'an River Basin, the Jiuzhou River Basin, and the Tingjiang-Hanjiang River Basin.

Collating the emission and reduction of pollution, we obtain the following evolutionary dynamics of pollution stock $s_{i}(t), i=1,2$, in upstream and downstream regions, respectively.

$$
\&(t)=q_{1}(t)-k_{1}(t)-u_{1}(t)-(\eta+\phi) s_{1}(t)
$$

$$
\stackrel{\&}{\&}(t)=q_{2}(t)-k_{2}(t)-u_{2}(t)+\phi s_{1}(t)-\eta s_{2}(t)
$$

where $s_{i}(0)=s_{i 0} \geq 0, \quad i=1,2$ are the initial stock of water pollutants in both regions, $0<\phi<1$ is the transfer coefficient of water pollutants transferred from the upstream region to the downstream region, and $\eta>0$ is the natural decay rate of water pollutants. We assume that there are identical natural decay rate for both regions.

Following Peng (2014) and Li et al. (2019), assume both regions suffer from the damage of linear stock pollution with marginal damage rate $\varepsilon$.

Taking the profit of Manufacturer 1, consumer surplus, tax revenue, pollution damage, and net government spending on pollution abatement into account constitutes the following social welfare function for the upstream region:

$$
H_{1}=(p(t)-c) q_{1}(t)-\frac{k_{1}^{2}(t)}{2}+\frac{b\left(q_{1}(t)+q_{2}(t)\right)^{2}}{4}-\varepsilon s_{1}(t)-\frac{(1-\delta) u_{1}^{2}(t)}{2}
$$

where by assuming the symmetry of consumer markets in the two regions, obtain the consumer surplus in each region $\frac{1}{2} * \frac{b\left(q_{1}(t)+q_{2}(t)\right)^{2}}{2}=\frac{b\left(q_{1}(t)+q_{2}(t)\right)^{2}}{4}$.

The upstream region's goal is to find the optimal pollution abatement investment and emission tax rate on the Manufacturer 1 to maximize its net present value of social welfare over the continuous time, $t, t \in 0, \infty)$ : 


$$
\&(t)=q_{1}(t)-k_{1}(t)-u_{1}(t)-(\eta+\phi) s_{1}(t)
$$

Similar to Equation 5, give the social welfare functions of the downstream region as:

$$
H_{2}=(p(t)-c) q_{2}(t)-\frac{k_{2}^{2}(t)}{2}+\frac{b\left(q_{1}(t)+q_{2}(t)\right)^{2}}{4}-\varepsilon s_{2}(t)-\frac{u_{2}^{2}(t)}{2}-\frac{\delta u_{1}^{2}(t)}{2}
$$

Therefore, the concern of the downstream region is:

$$
\max _{u_{2}, \tau_{2}, \delta} \int_{0}^{+\infty} e^{-\rho t} H_{2} d t
$$

$$
\left\{\begin{array}{l}
\&(t)=q_{1}(t)-k_{1}(t)-u_{1}(t)-(\eta+\phi) s_{1}(t) \\
\&(t)=q_{2}(t)-k_{2}(t)-u_{2}(t)+\phi s_{1}(t)-\eta s_{2}(t)
\end{array}\right.
$$

Having modeled the concerns of manufacturers and governments, as shown in Figure 2, we next section.

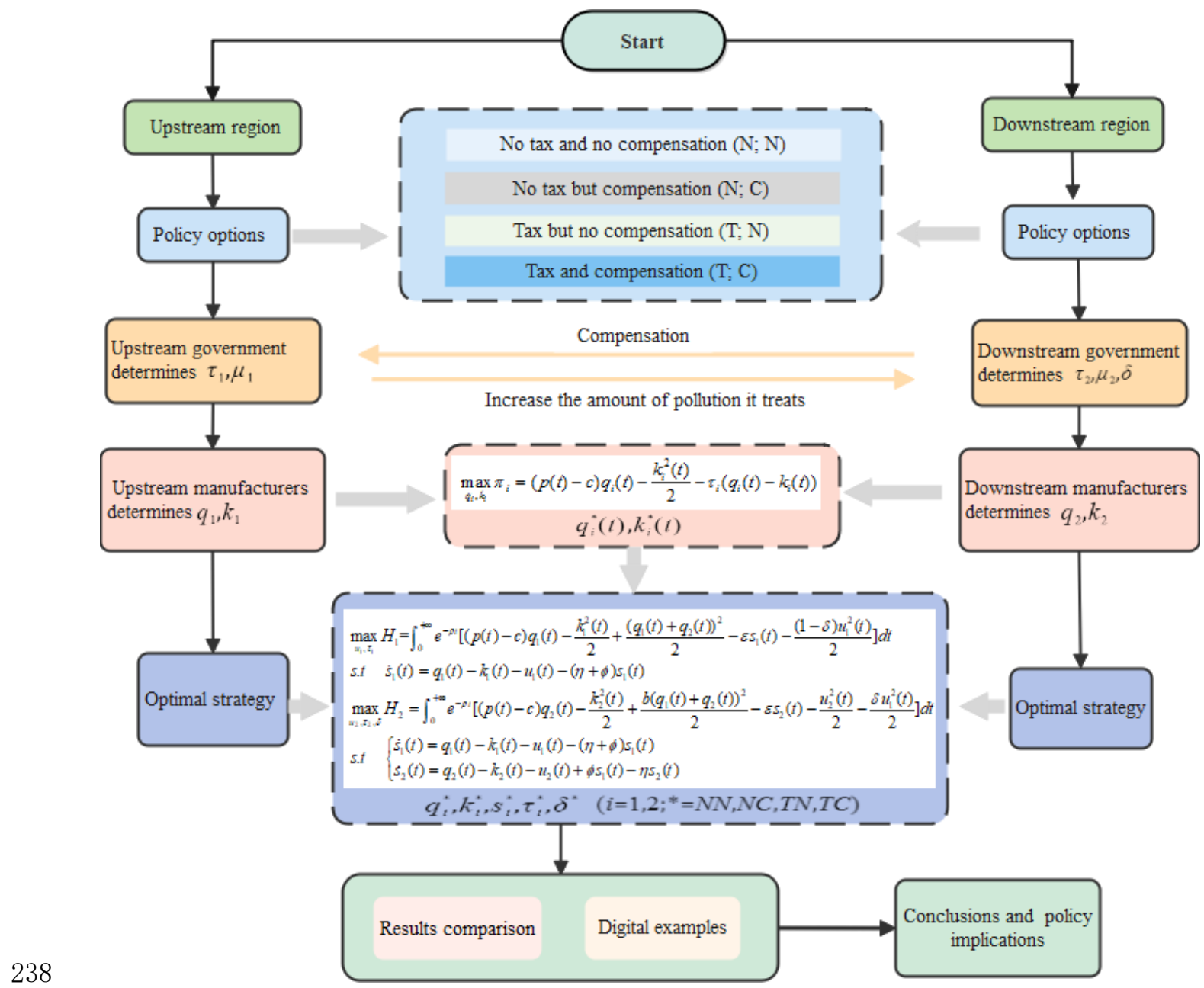




\section{Game Equilibrium}

Let us choose the combination strategy (no tax; no compensation) abbreviated as $(\mathrm{N}$; $\mathrm{N}$ ) to start the model analysis.

\subsection{Game equilibrium under no tax and no compensation $(\mathrm{N} ; \mathrm{N})$}

If both regions in the basin do not levy emissions taxes on the respective manufacturer, i.e., $\tau_{1}=\tau_{2}=0$, the manufacturers have no incentive to make pollution reductions. At the same time, if the downstream region does not compensate the upstream region for its investment in pollution control, i.e., $\delta=0$, the upstream region will not consider the interests of the downstream region to increase the pollution reduction. In this situation, we obtain the following steady-state equilibrium game result expressed in Theorem 1.

Theorem 1. Without government taxation on the manufacturers and compensation from the downstream region to the upstream region, the game has the following steady-state equilibrium results:

$$
k_{1}^{N N}=k_{2}^{N N}=0 ;
$$

$$
u_{1}^{N N}=\frac{\varepsilon}{(\eta+\phi+\rho)} ; u_{2}^{N N}=\frac{\varepsilon}{(\eta+\rho)} ;
$$

$$
s_{1}^{N N}=\frac{a-c}{3(\eta+\phi)}-\frac{\varepsilon}{(\eta+\phi+\rho)(\eta+\phi)}
$$

$$
s_{2}^{N N}=\frac{a-c}{3 \eta}+\frac{\phi(a-c)}{3 \eta(\eta+\phi)}-\frac{\varepsilon}{\eta(\eta+\rho)}-\frac{\varepsilon \phi}{\eta(\eta+\phi+\rho)(\eta+\phi)}
$$

$$
q_{1}^{N N}=q_{2}^{N N}=\frac{a-c}{3}
$$

$$
W_{1}^{N N}=\frac{(a-c)^{2}}{3}+\frac{\varepsilon^{2}}{(\eta+\phi)(\eta+\rho+\varphi)}-\frac{\varepsilon(a-c)}{3(\eta+\phi)}-\frac{\varepsilon^{2}}{2(\eta+\rho+\phi)^{2}}
$$

$$
W_{2}^{N N}=\frac{(a-c)^{2}}{3}+\frac{\varepsilon^{2} \phi}{\eta(\eta+\phi)(\eta+\rho+\phi)}+\frac{\varepsilon^{2}}{\eta(\eta+\rho)}-\frac{\varepsilon \phi(a-c)}{3 \eta(\eta+\phi)}-\frac{\varepsilon(a-c)}{3 \eta}-\frac{\varepsilon^{2}}{2(\eta+\rho)^{2}}
$$


$\delta^{N N}=0$

The proof is given in Appendix 1.

Theorem 1 describes the results in the state of complete absence of policy intervention. These results provide a benchmark for comparing the results in the state with policy intervention.

\subsection{Game equilibrium under no tax but compensation $(\mathrm{N} ; \mathrm{C})$}

This subsection investigates what the governments of both regions do not tax their manufacturers on emissions, but the downstream region compensates the upstream region for the pollution control investment. In this case, since the government does not regulate the manufacturer's emissions, the manufacturer does not automatically make the reduction. However, since pollution causes damage in each region, both upstream and downstream regional governments invest in pollution reduction. In particular, compensation from the downstream region comes with additional reduction investments in the upstream region. By game analysis, we give following Theorem 2.

Theorem 2. If local governments in the watershed do not tax on the manufacturers but implement ecological compensation, the game has the following steady-state equilibrium results:

$$
k_{1}^{N C}=k_{2}^{N C}=0
$$

$$
u_{1}^{N C}=\frac{\varepsilon+\varepsilon(\eta+\phi+\rho)}{2(\eta+\phi+\rho)^{2}} ; u_{2}^{N C}=\frac{\varepsilon}{(\eta+\rho)}
$$

$$
s_{1}^{N C}=\frac{(a-c)}{3(\eta+\phi)}-\frac{\varepsilon+\varepsilon(\eta+\phi+\rho)}{2 \eta(\eta+\phi+\rho)^{2}(\eta+\phi)}
$$

$$
s_{2}^{N C}=\frac{(a-c)}{3 \eta}-\frac{\varepsilon}{\eta(\eta+\rho)}+\frac{(a-c) \phi}{3 \eta(\eta+\phi)}-\frac{\varepsilon \phi+\varepsilon \phi(\eta+\phi+\rho)}{2 \eta(\eta+\phi+\rho)^{2}(\eta+\phi)}
$$

$$
\begin{aligned}
& q_{1}^{N C}=q_{2}^{N C}=\frac{a-c}{3} \\
& W_{1}^{N C}=\frac{(a-c)^{2}}{3}-\frac{\varepsilon(a-c)}{3(\eta+\phi)}+\frac{\varepsilon^{2}(\eta+\rho+\phi)+\varepsilon^{2}}{2(\eta+\phi)(\eta+\rho+\phi)^{2}}-\frac{\varepsilon^{2}(1+(\eta+\rho+\phi))}{4(\eta+\rho+\phi)^{3}}
\end{aligned}
$$




$$
W_{2}^{N C}=\frac{(a-c)^{2}}{3}+\frac{\varepsilon^{2}(\phi(\eta+\rho+\phi)+\phi)}{2 \eta(\eta+\phi)(\eta+\rho+\phi)^{2}}+\frac{\varepsilon^{2}}{\eta(\eta+\rho)}-\frac{\varepsilon(a-c)(\eta+2 \phi)}{3 \eta(\eta+\phi)}-\frac{\varepsilon^{2}}{2(\eta+\rho)^{2}}
$$

$$
-\frac{\varepsilon^{2}-\varepsilon^{2}(\eta+\rho+\phi)^{2}}{8(\eta+\rho+\phi)^{4}}
$$

$$
\tau_{1}^{N C}=\tau_{2}^{N C}=0
$$

$$
\delta^{N C}=\frac{1-(\eta+\phi+\rho)}{1+(\eta+\phi+\rho)}
$$

We put its proof in Appendix 2.

Comparing Theorem 1 and Theorem 2, one can see that, due to the implementation of ecological compensation, there are the following differences in the results under the two strategies described by the two Theorems 1 and 2:

(1) While pollution control investments in the downstream region remain unchanged, the investment in emission reduction in the upstream region increases by

$$
\frac{\varepsilon \delta}{(\eta+\phi+\rho)(1-\delta)}
$$

(2) The pollution stock in the upstream and downstream regions decreases, respectively

where $(1-\eta-\phi-\rho)>0$.

$$
\frac{\varepsilon \delta}{(\eta+\phi+\rho)(\eta+\phi)(1-\delta)} \text { and } \frac{\varepsilon \phi(1-\eta-\phi-\rho)}{2 \eta(\eta+\phi+\rho)^{2}(\eta+\phi)}
$$

\subsection{Game equilibrium under tax but no compensation ( $T$; $N$ )}

Now, we investigate what the local governments in the basin tax their manufacturers on emissions but do not implement ecological compensation policies. In this situation, the tax forces manufacturers to take responsibility for reducing emissions.

Solving the game model under (T; N), we reach the following Theorem 3.

Theorem 3. If local governments in the basin levy an emissions tax on their manufacturers but do not enforce ecological compensation, the game between the parties leads to the following equilibrium results:

$$
k_{1}^{T N}=\tau_{1}^{T N} ; k_{2}^{T N}=\tau_{2}^{T N}
$$




$$
u_{1}^{T N}=\frac{\varepsilon}{(\eta+\phi+\rho)} ; u_{2}^{T N}=\frac{\varepsilon}{(\eta+\rho)}
$$

$$
s_{1}^{T N}=\frac{13(a-c)}{12(\eta+\phi)}-\frac{31 \varepsilon}{6(\eta+\phi+\rho)(\eta+\phi)}+\frac{(5 \eta+4 \phi) \varepsilon}{12(\eta+\phi)^{2}(\eta+\phi)}
$$

$$
s_{2}^{T N}=\frac{7(a-c)}{12 \eta}+\frac{(5 \eta+4 \phi) \varepsilon \phi}{12 \eta(\eta+\phi)^{2}(\eta+\rho)}+\frac{5 \varepsilon(\eta+\phi)-31 \varepsilon \phi}{6 \eta(\eta+\phi+\rho)(\eta+\phi)}
$$

$$
-\frac{(37 \eta+32 \phi) \varepsilon-13 \phi(a-c)(\eta+\rho)}{12 \eta(\eta+\phi)(\eta+\rho)}
$$

$$
q_{1}^{T N}=\frac{7(a-c)}{12}+\frac{(5 \eta+4 \phi) \varepsilon}{12(\eta+\phi)(\eta+\rho)}-\frac{5 \varepsilon}{3(\eta+\phi+\rho)}
$$

$$
q_{2}^{T N}=\frac{a-c}{3}+\frac{5 \varepsilon}{6(\eta+\phi+\rho)}-\frac{(5 \eta+4 \phi) \varepsilon}{6(\eta+\phi)(\eta+\rho)}
$$

$$
W_{1}^{T N}=\frac{(a-c)^{2}}{2}+\frac{62 \varepsilon^{2}}{12(\eta+\phi)(\eta+\rho+\phi)}+\frac{1}{96}\left(\frac{\varepsilon(5 \eta+4 \phi)}{(\eta+\rho)(\eta+\phi)}-(a-c)\right)^{2}-\frac{5 \varepsilon(a-c)}{6(\eta+\rho+\phi)}
$$

$$
-\frac{1}{6}\left(\frac{5 \varepsilon}{(\eta+\rho+\phi)}-(a-c)\right)^{2}-\frac{13 \varepsilon(a-c)}{12(\eta+\phi)}-\frac{\varepsilon^{2}(5 \eta+4 \phi)}{12(\eta+\phi)^{2}(\eta+\rho)}-\frac{\varepsilon^{2}}{2(\eta+\rho+\phi)^{2}}
$$

$$
\begin{aligned}
& W_{2}^{T N}=\frac{5(a-c)^{2}}{12}+\frac{31 \varepsilon^{2} \phi-5 \varepsilon^{2}(\eta+\phi)}{6 \eta(\eta+\phi)(\eta+\rho+\phi)}+\frac{1}{24}\left(\frac{5 \varepsilon}{\eta+\rho+\phi}-(a-c)\right)^{2} \\
& -\frac{1}{24}\left(\frac{\varepsilon(5 \eta+4 \phi)}{(\eta+\rho)(\eta+\phi)}-(a-c)\right)^{2}-\frac{\varepsilon^{2} \phi(5 \eta+4 \phi)}{12 \eta(\eta+\rho)(\eta+\phi)^{2}}-\frac{\varepsilon^{2}}{2(\eta+\rho)^{2}}
\end{aligned}
$$

$$
-\frac{\varepsilon(a-c)(5 \eta+4 \phi)+\varepsilon(a-c)(7 \eta+20 \phi)(\eta+\rho)-\varepsilon^{2}(37 \eta+32 \phi)}{12 \eta(\eta+\rho)(\eta+\phi)}
$$

$$
\tau_{1}^{T N}=\frac{5 \varepsilon}{2(\eta+\phi+\rho)}-\frac{(a-c)}{2} ; \tau_{2}^{T N}=\frac{(5 \eta+4 \phi) \varepsilon}{4(\eta+\phi)(\eta+\rho)}-\frac{(a-c)}{4}
$$

$$
\delta^{T N}=0
$$

The proof of Theorem 3 can be found in Appendix 3.

From Theorem 3, one can see that the real incentive for manufacturers to make investments in emissions reductions is the emissions tax, without a tax on emissions, a manufacturer's investment in pollution control equals zero.

\subsection{Game equilibrium under tax and compensation ( $T$; $C$ )}

If both emissions tax and ecological compensation policies are applied, manufacturers and 
local governments in the basin will be fully motivated to reduce emissions. Therefore, taking these two policies into account, we obtain the multiple game result described in Theorem 4.

Theorem 4. If local governments in the basin levy an emissions tax on their manufacturers as well as enforce ecological compensation, the game results in the following steady-state equilibrium results:

$$
\begin{aligned}
& k_{1}^{T C}=\tau_{1}^{T C} ; k_{2}^{T C}=\tau_{2}^{T C} \\
& u_{1}^{T C}=\frac{\varepsilon+\varepsilon(\eta+\phi+\rho)}{2(\eta+\phi+\rho)^{2}} ; u_{2}^{T C}=\frac{\varepsilon}{(\eta+\rho)}
\end{aligned}
$$

$$
s_{1}^{T C}=\frac{13(a-c)}{12(\eta+\phi)}-\frac{25 \varepsilon}{6(\eta+\phi+\rho)(\eta+\phi)}+\frac{(5 \eta+4 \phi) \varepsilon}{12(\eta+\phi)^{2}(\eta+\rho)}-\frac{\varepsilon+\varepsilon(\eta+\phi+\rho)}{2(\eta+\phi+\rho)^{2}(\eta+\varphi)}
$$

$$
s_{2}^{T C}=\frac{7(a-c)}{12 \eta}+\frac{5 \varepsilon(\eta+\phi)}{6 \eta(\eta+\phi+\rho)(\eta+\phi)}-\frac{\varepsilon(37 \eta+32 \varphi)-13 \phi(a-c)(\eta+\rho)}{12 \eta(\eta+\phi)(\eta+\rho)}+\frac{(5 \eta+4 \phi) \varepsilon \phi}{12 \eta(\eta+\phi)^{2}(\eta+\rho)}
$$

$$
-\frac{3 \varepsilon \phi+28 \varepsilon \phi(\eta+\phi+\rho)}{6 \eta(\eta+\phi+\rho)^{2}(\eta+\phi)}
$$

$$
q_{1}^{T C}=\frac{7(a-c)}{12}+\frac{(5 \eta+4 \phi) \varepsilon}{12(\eta+\phi)(\eta+\rho)}-\frac{5 \varepsilon}{3(\eta+\phi+\rho)}
$$

$$
q_{2}^{T C}=\frac{a-c}{3}+\frac{5 \varepsilon}{6(\eta+\phi+\rho)}-\frac{(5 \eta+4 \phi) \varepsilon}{6(\eta+\phi)(\eta+\rho)}
$$

$$
W_{1}^{T C}=\frac{(a-c)^{2}}{2}+\frac{28 \varepsilon^{2}(\eta+\rho+\phi)+3 \varepsilon^{2}}{6(\eta+\phi)(\eta+\rho+\phi)^{2}}+\frac{1}{6}\left(\frac{c-a}{4}+\frac{\varepsilon(5 \eta+4 \phi)}{4(\eta+\rho)(\eta+\phi)}\right)^{2}-\frac{2}{3}\left(\frac{c-a}{2}+\frac{5 \varepsilon}{2(\eta+\rho+\phi)}\right)^{2}
$$

$$
-\frac{13(a-c)}{12(\eta+\phi)}-\frac{\varepsilon^{2}(5 \eta+4 \phi)}{12(\eta+\rho)(\eta+\phi)^{2}}-\frac{2 \varepsilon^{2}(\eta+\rho+\phi)+2 \varepsilon^{2}}{8(\eta+\rho+\phi)^{3}}-\frac{5 \varepsilon(a-c)}{6(\eta+\rho+\phi)}
$$

$$
\begin{aligned}
& W_{2}^{T C}=\frac{5(a-c)^{2}}{12}+\frac{\varepsilon^{2}(37 \eta+32 \phi)}{12 \eta(\eta+\rho)(\eta+\phi)}+\frac{\varepsilon^{2}(23 \phi-5 \eta)(\eta+\rho+\phi)+3 \varepsilon^{2} \phi}{6 \eta(\eta+\phi)(\eta+\rho+\phi)^{2}} \\
& +\frac{1}{6}\left(\frac{5 \varepsilon}{2(\eta+\rho+\phi)}-\frac{a-c}{2}\right)^{2}-\frac{\varepsilon^{2}}{2(\eta+\rho)^{2}}-\frac{\varepsilon(a-c)(7 \eta+20 \phi)}{12 \eta(\eta+\phi)}-\frac{\varepsilon^{2} \phi(5 \eta+4 \phi)}{12 \eta(\eta+\rho)(\eta+\phi)^{2}}
\end{aligned}
$$$$
-\frac{\varepsilon^{2}\left(1-(\eta+\rho+\phi)^{2}\right)}{8(\eta+\rho+\phi)^{4}}-\frac{\varepsilon(a-c)(5 \eta+4 \phi)}{12(\eta+\rho)(\eta+\phi)}-\frac{2}{3}\left(\frac{\varepsilon(5 \eta+4 \phi)}{4(\eta+\rho)(\eta+\phi)}-\frac{a-c}{4}\right)^{2}
$$

$$
\tau_{1}^{T C}=\frac{5 \varepsilon}{2(\eta+\phi+\rho)}-\frac{(a-c)}{2} ; \tau_{2}^{T C}=\frac{(5 \eta+4 \phi) \varepsilon}{4(\eta+\phi)(\eta+\rho)}-\frac{(a-c)}{4}
$$




$$
\delta^{T C}=\frac{1-(\eta+\phi+\rho)}{1+(\eta+\phi+\rho)}
$$

The proof of Theorem 4 can be found in Appendix 4 .

Through game analysis, we have obtained the steady-state equilibrium results under the four different policy combinations. Next, let us compare and analyze these results to obtain policy implications of the research results.

\subsection{Comparative analysis of the impact under different policy portfolios}

Comparing and analyzing the equilibrium results of the game under four different policy combinations, obtain the following inferences.

\subsubsection{Taxation strategy and ecological compensation strategy}

Below we use Corollary 1 and Corollary 2 to compare and analyze the government's taxation strategy and ecological compensation strategy.

Corollary 1.If both regions in the basin tax their manufacturers with an emission tax, but can optionally implement ecological compensation. The optimal tax rate for the upstream region and the downstream region is equal in both cases with and without compensation. In addition, the emission tax rate in the downstream region is higher than that in the upstream region, with or without ecological compensation. I.e.,

$$
\tau_{1}^{T N}=\tau_{1}^{T C}<\tau_{2}^{T N}=\tau_{2}^{T C}
$$

Because with or without ecological compensation, the downstream region must bear the pollutants from the upstream region. Therefore, it must levy a higher emission tax than the upstream region; From Corollary 1, one can find that ecological compensation does not influence the emissions tax decisions region in both regions.

Corollary 2. The ecological compensation rate is equal in both cases where both regions tax and do not tax their manufacturers simultaneously. I.e.,

$$
\delta^{T C}=\delta^{N C}=\frac{1-(\eta+\phi+\rho)}{1+(\eta+\phi+\rho)} .
$$

We see from Corollary 2 that the ecological compensation rate is independent of the manufacturer's output decision parameters, so the government's tax strategy has no effect on the 
ecological compensation rate.

We put the proof of Corollary 1 and Corollary 2 in Appendix 5.

\subsubsection{Manufacturers and the governments' pollution reduction}

Examining the reduction of manufacturer pollution in the two regions under the four scenarios, obtain the following Corollary3:

Corollary3. If the governments do not tax manufacturers on emissions, manufacturers do not make pollution reductions. However, if the governments levy an emissions tax, the pollution reduction rate of manufacturers in both regions is equal to the emissions tax rate, and whether or not implementing ecological compensation does not affect the manufacturers' pollution reduction rate. I.e.,

$$
k_{1}^{N N}=k_{1}^{N C}=k_{2}^{N C}=k_{2}^{N N}=0, k_{1}^{T N}=k_{1}^{T C}<k_{2}^{T N}=k_{2}^{T C} .
$$

If there is no tax on emissions, there is no cost to the manufacturer for emissions, but there is a cost to reduce emissions. So without a tax on emissions, manufacturers will not reduce emissions. However, if the governments levy an emission tax, there is a cost to emissions, and the higher the tax rate, the higher the cost of emissions. So the rate of pollution reduction by manufacturers is synchronized with the change in the emissions tax rate. Moreover, because Corollary 1 shows $\tau_{1}^{T N}=\tau_{1}^{T C}<\tau_{2}^{T N}=\tau_{2}^{T C}$, there for, we have $k_{1}^{T N}=k_{1}^{T C}<k_{2}^{T N}=k_{2}^{T C}$.

Now, let us compare the pollution control investments of the two regional governments and by which we give the following corollary 4 :

Corollary4. The amount of pollution control investment by governments in the upstream and downstream regions is independent of the emissions tax policy, and the treatment investment in the downstream region is independent of the ecological compensation policy. Ecological compensation makes the upstream region increase its pollution control investments. Nevertheless, with or without implementing eco-compensation, the pollution control investment of the upstream region government is lower than that of the downstream region government. I.e., $u_{2}^{T C}=u_{2}^{T N}=u_{2}^{N C}=u_{2}^{N N}=u_{2}, u_{1}^{T N}=u_{1}^{N N}<u_{1}^{T C}=u_{1}^{N C}<u_{2}$.

First, since the downstream region faces two sources of pollution, i.e., the upstream and downstream manufacturers, the upstream region faces only one source of pollution from the upstream manufacturer. Therefore, the downstream region needs to make more investment in 
pollution control. Second, ecological compensation can increase the total pollution control investment of the governments in the watershed, which is the same as Yi et al. (2020a). However, unlike Yi et al. (2020a), ecological compensation does not shift pollution control investment downstream to upstream regions but only increases investment in the upstream region. It may be because this paper adds the consideration of government regulation on manufacturers' pollution behavior.

The proof of Corollary 3 and Corollary 4 can be found in Appendix 6 . Corollary 5:

\subsubsection{Pollution stock}

This subsection compares the pollutant stocks in the three cases and we get following

Corollary 5. The pollution stock in both regions decreases with the implementation of (no tax; no compensation), (no tax; compensation), (tax; no compensation), and (taxation; compensation) four policy combinations. Moreover, under the four policies, the pollution stock in the downstream region is always higher than in the upstream region. I.e.,

$$
\begin{aligned}
& \quad \mathbf{s}_{1}^{T C}<\mathbf{s}_{1}^{T N}<\mathbf{s}_{1}^{N C}<\mathbf{S}_{1}^{N N}, \mathbf{s}_{2}^{T C}<\mathbf{s}_{2}^{T N}<\mathbf{S}_{2}^{N C}<\mathbf{S}_{2}^{N N} ; \mathbf{s}_{1}^{N N}<\mathbf{S}_{2}^{N N}, \mathbf{S}_{1}^{N C}<\mathbf{s}_{2}^{N C}, \mathbf{s}_{1}^{T C}<\mathbf{s}_{2}^{T C}, \\
& \mathbf{s}_{1}^{T N}<\mathbf{S}_{2}^{T N} .
\end{aligned}
$$

The proof of Corollary 5 can be found in Appendix 7.

From Corollary 5, one can find the importance of local governments choosing appropriate environmental management policies in a watershed. In addition, since the downstream region faces two sources of pollution, the upstream and downstream manufacturers, the pollution stock is always higher than in the upstream region, which faces only one source of pollution from the upstream manufacturer.

\subsubsection{Production and social welfare}

Finally, let us compare the output and social welfare of the two regions under different policy combinations.

Analyzing the optimal output of the two regions, we obtain the following Corollary 6 :

Corollary 6. Ecological compensation has no effect on production in both regions, and the production in the two regions is equal when the governments of the two regions do not impose an emissions tax on manufacturers. However, if the governments in both regions impose an emissions 
tax, the production of the manufacturer in the downstream region declines, while the manufacturer's production in the upstream region increases. I.e.,

$$
\begin{aligned}
& q_{1}^{N N}=q_{1}^{N C}=q_{2}^{N N}=q_{2}^{N C}=(a-c) / 3, \quad q_{1}^{T N}=q_{1}^{T C}>q_{1}^{N N}=q_{1}^{N C}, q_{1}^{T C}>q_{2}^{T C}, q_{1}^{T N}>q_{2}^{T N}, \\
& q_{2}^{N N}=q_{2}^{N C}>q_{2}^{T N}=q_{2}^{T C} .
\end{aligned}
$$

Without the emissions tax, the manufacturers would compete each other in the Cournot market without interference by the government, so they all produce a Cournot volume. However, if the governments in both regions impose an emissions tax, the tax puts downward pressure on the manufacturers' output because the tax increases marginal costs in both regions. However, as shown in Corollary 1, the tax rate in the upstream region is lower than it in the downstream region, making the taxed marginal cost of the manufacturer in the upstream region lower than it in the downstream region, and this difference in marginal cost causes the output of the manufacturer in the upstream region to rise while the output of the manufacturer in the downstream region to fall further. Interestingly, for the manufacture in the upstream region, the impact on the output of the marginal cost difference between the two regions exceeds the impact on the output of the marginal cost increase so that, ultimately, its output ends up being higher if the governments levy an emissions tax in both regions.

Next, we use Corollary 7 to compare and analyze the social welfare of the two regions.

Corollary 7. For the both upstream and downstream regions, from lowest to highest, the ranking of the lever of social welfare under the four policies is (no tax; no compensation), (no tax; compensation), (tax; no compensation), and (taxation; compensation). In addition, the level of welfare in the downstream region is consistently lower than it in the upstream region. I.e., $W_{1}^{N N}<W_{1}^{N C}<W_{1}^{T N}<W_{1}^{T C}, W_{2}^{N N}<W_{2}^{N C}<W_{2}^{T C}<W_{2}^{T N}, W_{2}^{N C}<W_{1}^{N C}, W_{2}^{N N}<W_{1}^{N N}$.

The proof of Corollary 6 and Corollary 7 can be see in Appendix 8.

Since in this paper, we assume that the manufacturers in the two regions are symmetric. However, the downstream region is subject to pollution from manufacturers in the upstream and downstream regions, while the upstream region is subject to pollution from manufacturers in the upstream region only. Therefore, the welfare level in the upstream region is higher. Moreover, as shown by Yi et al. (2021), ecological compensation can lead to a Pareto improvement because: on the one hand, the welfare level of the compensation fund provider, the downstream region, will 
not reduce. Otherwise, it would not provide compensation; on the other hand, in the upstream region, the recipient of compensation funds can use funds from the downstream region to improve its water environment, and its welfare level will undoubtedly increase. Therefore, ecological compensation in a watershed will increase the welfare of at least one party and not reduce the welfare of any party. Of course, in addition to curbing pollution, the emissions tax is also a tool to raise social welfare. Otherwise, the governments would not use it. Therefore, the simultaneous use of the two policies leads to the highest level of social welfare in both regions.

\subsection{Policy implications}

At this point, we have come to the clear conclusion that the water environment in the entire basin is cleanest, and the level of social welfare in each region is highest under the combined (taxation; compensation) policy. Let us analyze the reasons for this result: (1) If there is neither taxation nor ecological compensation, manufacturers will emit more regardless of the pollution externalities. In addition, the upstream region will only consider the impact of pollution on itself and not on the downstream, and thus the amount of treatment is less than the socially optimal one. Herefore, (no taxation; no compensation) leaves the externalities completely uninternalized, leading to the lowest economic efficiency and the worst environmental pollution. (2) The portfolio policy (taxation; no compensation) can internalize the externalities caused by manufacturers, but it cannot play the incentive of pollution control in the upstream region. (3) The portfolio policy (no taxation; compensation) makes the upstream region increase investment in treatment due to the compensation of the downstream region, but the manufacturer will emit more regardless of the external influences. (4) Under the portfolio policy (taxation; compensation), on the one hand, the tax internalizes the externalities of manufacturers, thus producing the socially optimal output and improving both social welfare and the environment due to the reduction of pollution by manufacturers. On the other hand, obtaining the compensations from the downstream region, the upstream region will increase its treatment investments, resulting in a cleaner water environment for the entire basin. At the same time, to some extent, the shift of the investment from downstream to upstream can increase the marginal benefit of investment because pollution control in the upstream can clean the entire basin while pollution control in the downstream can only clean the downstream. As studied by Yi et al. (2021), ecological compensation can lead to a Pareto 
improvement. Therefore, from ecological improvement and economic efficiency, the combination policy of taxation and compensation is a wise choice for the local governments in a watershed.

Realistically, the Chinese government started to implement the Environmental Protection Tax Law of the People's Republic of China in 2018, which changed the management of pollution emissions from the original fee system to a tax on emissions. China's environmental situation has continued to improve since implementing policy measures such as the emissions tax. Data from China's ecological environment status bulletin shows that in 2017, 99 of China's 338 large and medium-sized cities meet air quality standards, accounting for $29.3 \%$ of the total. The annual average concentration of PM 2.5 is $43 \mu \mathrm{g} / \mathrm{m}^{3}$. Of the 1617 major river water quality cross-sections, $71.8 \%$ are of category I-III quality, and $8.4 \%$ are of category V quality. However, in 2020, among the 337 large and medium-sized cities in China, the number of cities meeting air quality standards is 202 , accounting for $59.9 \%$, and the average proportion of good air quality days is $87 \%$. The annual average concentration of PM 2.5 is $33 \mu \mathrm{g} / \mathrm{m}^{3}$. Among the 1614 major river water quality sections, $77.4 \%$ of the I-III water quality sections and only $0.2 \%$ of the category V quality ${ }^{1}$.

Along with implementing the emissions tax system, the Chinese government has actively promoted ecological compensation in significant watersheds and has achieved good results. For example, in China's Jiuzhou River basin, the upstream of Guangxi Province and the downstream of which is Guangdong Province. At the junction of the two provinces, the water quality at the "Wen Che Qiao" section is category V before implementing ecological compensation. However, since the implementation of ecological compensation in 2014 , the water quality in this section has reached category III. A similar situation also occurred in the Xin'an River and other watersheds that have implemented ecological compensation. ${ }^{2}$

Thus, one can see that China's policy of combining emissions tax and ecological compensation in watershed ecological management has achieved good results.

\section{Conclusions}

\footnotetext{
${ }^{1}$ https://www. mee. gov. cn/

${ }^{2}$ https://news. ifeng. com/c/7fcW04GBqN7
} 
China and other countries worldwide have widely used a combination of emissions tax and ecological compensation policy for watershed environmental management. However, the academic community has rarely shed light on it so far. Therefore, it is not conducive to the optimal design of the current policy or the timely adjustment of the policy to the changing situation. With this in mind, this paper constructs a multiple game model to analyze product market competition and ecological compensation games among watershed regions. We assume that the upstream and downstream regions of the basin are free to choose (no tax, no compensation), (no tax, compensate), (tax, no compensation), and (tax, compensate) four strategies to regulate the manufacturer while cooperating with other regions of the basin. By solving this multiple game model, we obtain the simultaneous equilibrium between the governments and manufacturers in the four cases for the first time. In particular, we obtain the following key conclusions with great managerial significance:

First, the (tax, compensation), (tax, no compensation), (no tax, compensation), and (no tax, no compensation) combination policies result in the highest, second, third, and lowest social welfare and water ecology, respectively, for all regions in basin.

Second, government investment in environmental management in the downstream region is equal in all four cases, and ecological compensation does not shift investment from downstream to upstream, but it can induce the upstream region to increase investment in management.

Third, suppose the governments impose an emission tax, the tax rate in the downstream region is higher than that in the upstream region. Moreover, the tax rate in the upstream region is equal with and without ecological compensation, while the tax rate in the downstream region is higher under ecological compensation; Furthermore, manufacturers invest in treatment at the same rate as the tax rate in their regions.

Fourth, if there is no tax on emissions, with or without ecological compensation, the manufacturers' output in the two regions is equal, both equal to one-third of the market capacity. Moreover, all the manufacturers do not perform pollution control. However, if the governments impose an emissions tax, manufacturers' output in both regions decreases, and it in the upstream region is higher than it in the downstream region. In addition, the downstream manufacturer invests more in pollution control than the upstream manufacturer.

Finally, ecological compensation rate independent of emissions tax policy, tax or not does not 
affect the ecological compensation rate.

Our study has yielded some clear conclusions about several different environmental management policies in a basin. Nevertheless, more importantly, our study provides an analytical framework for designing an optimal emissions tax and ecological compensation joint policy mechanism. Therefore, local governments in a basin can follow our framework to design optimal environmental management policies and adjust the emissions tax rate and ecological compensation rate according to the changing parameters, making the policy objectives always aimed at the socially optimal welfare level.

Also, it should be noted that there are several problems in this paper that we expect to solve in the future. First, this paper assumes that the damage function of pollution to the environment is linear. However, a nonlinear environmental damage function may be more realistic. Second, there may be technology spillover in pollution controls investment, and this spillover may influence the behavior of the local governments and manufacturers, but this paper does not analyze it. Third, this paper does not consider the uncertainties that may exist in the evolution of the pollution stock. We will do further research to address the above issues in the future.

\section{Authors' contributions}

Yi Yongxi focuses on the conceptualization, methodology, writing original draft, Writing-review \& editing. Ding Caini focuses on the calculation, formal analysis. Fu Chunyan focuses on the validation and writing-original draft. Li Yuqiong focuses on methodology, writing-original draft.

\section{Funding}

The study and collection, analysis, and interpretation of data were supported by the National Natural Science Foundation of China (71970491) and the Hunan Provincial Social Science Outcomes Review Committee Major Projects (No. XSP20ZDA007)

\section{Availability of data and materials}

All data generated or analysed during this study are included in this published article

\section{Compliance with ethical standards}

\section{Ethics approval}

Not applicable 


\section{Consent to Participate}

Not applicable

\section{Consent to Publish}

\section{Competing interests}

The authors declare that they have no competing interests

\section{References}

Barnett, A.H., 1980. The Pigouvian tax rule under monopoly. Am. Econ. Rev. 70 (5), 1037-1104.

Boucekkine, R., Fabbri, G., Federico, S., Gozzi, F., 2021. From firm to global-level pollution control: The case of transboundary pollution. Eur. J. Oper. Res. 290, 331-345.

Breton, M., Sokri, A., Zaccour, G., 2008. Incentive equilibrium in an overlapping- generations environmental game. Eur. J. Oper. Res. 185 (2), 687-699.

Buchanan, J M, External Diseconomies, 1969. Corrective Taxes, and Market Structure. Am Econ Rev, 59 (1), 174-177.

Carraro, C. and Siniscalco, D., 1993. Strategies for the Protection of the Environment. J Public Econ, 52, 309-328.

Chen, W.T., Hu, Z.H., 2018. Using evolutionary game theory to study governments and manufacturers' behavioral strategies under various carbon taxes and subsidies. J. Clean. Prod, $148,123-141$.

Cranford, M., Mourato,S., 2011. Community conservation and a two-stage approach to payments for ecosystem services. Ecol Econ. 71, 89-98.

De Frutos J , López, Paula, Martín-Herrán, Guiomar.,2021. Equilibrium strategies in a multiregional transboundary pollution differential game with spatially distributed controls. Automatica.125, 109411.

Dockner, E.J., Long, N.V., 1993. International pollution control: cooperative versus noncooperative strategies. J. Environ. Econ. Manag. 25, 13-29

Feichtinger, G., Lambertini, L., George Leitmann, G., Wrzaczek, S., 2016. R\&D for green technologies in a dynamic oligopoly: Schumpeter, arrow and inverted-U' s. Eur. J. Oper. Res. 
249, 1131-1138.

600

601

602

603

604

605

606

607

608

609

610

611

612

613

614

Gao, X., Shen,J., He, W., et al.,2019. An evolutionary game analysis of governments' decision-making behaviors and factors influencing watershed ecological compensation in China. J Environ Manage. 251, Article ID 109592.

Huang, X., He, P., Zhang, W., 2016. A cooperative differential game of transboundary industrial pollution between two regions. J. Clean. Prod. 120, 43-52.

Jiang, K., You, D.M., Li, Z,D., Shi, S.S., 2019. A differential game approach to dynamic optimal control strategies for watershed pollution across regional boundaries under eco-compensation criterion. Ecol. Indic. 105,229-241.

Jiao, Y.Q., Su, M.R., Ji, C.W., Yang, S.Y., Zhang, P., 2021. How to design fully cooperative policies to abate transboundary air pollution between two highly asymmetric regions: An abnormal incrementalism analysis. J. Clean. Prod. 278, 124042.

Kemkes, R. J., Farley, J., Koliba, C. J., 2010. Determining when payments are an effective policy approach to ecosystem service provision. Ecol Econ. 69(11), 2069-2074.

Lambertini, L., Poyago-Theotoky, J., Tampieri, A., 2017. Cournot competition and "green" innovation: an inverted-U relationship. Energy Econ, 68, 116-123.

Li, H, U., Guo, G. L., 2019. A differential game analysis of multipollutant transboundary pollution in river basin. Physica A. 535,122484.

Long, N.V., 1992. Pollution control: a differential game approach, Ann. Oper. Res. 37, 283-296.

Lu, H.B., Li, J.K., Xiao, B., Guo, M., 2020. Analysis of standard accounting method of economic compensation for ecological pollution in watershed. Sci. Total Environ. 737, 138157.

MartínHerrán, Guiomar, Rubio S J., 2018. Second-best taxation for a polluting monopoly with abatement investment. Energy Econ, 73 (6), 178-193.

Muradian, R., Corbera, E., Pascual, U., et al., 2010. Reconciling theory and practice: an alternative conceptual framework for understanding payments for environmental services. Ecol Econ. 69(6), 1202-1208.

Peng, P., Zhang, J., 2014. Research for trans-boundary water pollution cooperation. Adv Mat Res, $864,1525-1530$.

Pigou, A.C. The Economics of Welfare. London:Macmillan,1920.

Ploeg, F. Van der., Zeeuw, Aart de., 1992. International aspects of pollution control, Environ. 
Shen, J.Q., Gao, X., He, W.J., Sun, F.H., Zhang, Z.F., 2021.Prospect theory in an evolutionary game: Construction of watershed ecological compensation system in Taihu Lake Basin. J. Clean. Prod. 291, 125929.

Wei, Z.J., Yi, Y.X., Fu, C.Y., 2019. Cournot competition and "green" innovation under efficiency-improving learning by doing. Physica A 531 (10), 121762.

Xepapadeas, A., 1992. Environmental policy design and dynamic non-point source pollution. J. Environ. Econ. Manag. 23, 22-39.

Yeung, D.W.K., 2007. Dynamically consistent cooperative solution in a differential game of transboundary industrial pollution. J. Optim. Theory Appl.134, 143-160.

Yi, Y., Wei, Z., \& Fu, C.. 2020b. An optimal combination of emissions tax and green innovation subsidies for polluting oligopolies. J. Clean. Prod, 284(3), 124693.

Yi, Y.X., Wei, Z.J., Fu, C.Y., 2020a., A differential game of transboundary pollution control and ecological compensation in a river basin. Complexity, 2020, 6750805.

Yi, Y.X., Yang, M., Fu, C.Y., 2021., Analysis of multiple ecological compensation strategies for transboundary pollution control in a river basin. Manage Decis Econ, 2021, 1-11.

Zeng, Y., Li,J., Cai,Y., Tan,Q., Dai, C., 2019. A hybrid game theory and mathematical 647 


\section{Appendix}

653

\section{Appendix 1}

Using (1) and (2), the manufacturers optimization problem can be given as follows:

$$
\frac{\partial \pi_{1}}{\partial q_{1}}=a-c-2 q_{1}-q_{2}=0 ; \frac{\partial \pi_{2}}{\partial q_{2}}=a-c-q_{1}-2 q_{2}=0
$$

$$
\frac{\partial \pi_{1}}{\partial k_{1}(t)}=k_{1}(t)=0 ; \frac{\partial \pi_{2}}{\partial k_{2}(t)}=k_{2}(t)=0
$$

Now, combining Eq.(5) and (6), we can get the current value Hamiltonian function of the upstream region :

$$
H_{1}\left(u_{1}, s_{1}\right)=\frac{a-c+2(a-c)^{2}}{9}-\varepsilon s_{1}(t)-\frac{u_{1}^{2}(t)}{2}+\lambda_{1}(t)\left(\frac{a-c}{3}-u_{1}(t)-(\eta+\phi) s_{1}(t)\right)
$$

$$
\frac{\partial H_{1}}{\partial u_{1}(t)}=-u_{1}(t)-\lambda_{1}(t)=0
$$

$$
k_{1}^{\prime}(t)=\rho \lambda_{1}(t)-\frac{\partial H_{1}}{\partial s_{1}(t)}=\varepsilon+(\eta+\phi+\rho) \lambda_{1}(t)
$$

$\operatorname{lof}_{1}(t)=-\not{\alpha}(t)=-\varepsilon+(\eta+\phi+\rho) u_{1}(t)$

Then, we obtain the following simultaneous differential equations:

$$
\left\{\begin{array}{l}
\&(t)=q_{1}(t)-u_{1}(t)-(\eta+\phi) s_{1}(t) \\
u_{\alpha}(t)=-\varepsilon+(\eta+\phi+\rho) u_{1}(t) \\
\alpha_{1}^{\&}(t)=\varepsilon+(\eta+\phi+\rho) \lambda_{1}(t)
\end{array}\right.
$$

Using the steady-state equilibrium condition $f(t)=L_{4} \&(t)=\not_{1}(t)=0$, The optimization results of the upstream region can be obtained by taking the partial derivatives of the relevant variables.

Next, combining Eq.(7) and (8), we can get the downstream region current value Hamiltonian function :

$$
\begin{aligned}
H_{2}\left(u_{2}, s_{2}\right)= & \frac{a-c+2(a-c)^{2}}{9}-\varepsilon s_{2}(t)-\frac{u_{2}^{2}(t)}{2}+\lambda_{2}(t)\left(\frac{a-c}{3}-u_{2}(t)+\phi s_{1}(t)-\eta s_{2}(t)\right) \\
& \left.+\lambda_{3}(t)\left(\frac{a-c}{3}-u_{1}(t)-(\eta+\varphi) s_{1}(t)\right)\right)
\end{aligned}
$$

$$
\frac{\partial H_{2}}{\partial u_{2}(t)}=-u_{2}(t)-\lambda_{2}(t)=0
$$

$$
d_{2}^{\&}(t)=\rho \lambda_{2}(t)-\frac{\partial H_{2}}{\partial s_{2}(t)}=\varepsilon+(\eta+\rho) \lambda_{2}(t)
$$


$\alpha_{3}(t)=\rho \lambda_{3}(t)-\frac{\partial H}{\partial s_{1}(t)}=(\rho+\eta+\varphi) \lambda_{3}(t)-\varphi \lambda_{2}(t)$

$u_{2}(t)=-\mathbb{k}_{2}(t)=-\varepsilon+(\eta+\rho) u_{2}(t)$

Then, we obtain the following simultaneous differential equations:

$$
\left\{\begin{array}{l}
\underset{\&}{\&}(t)=q_{1}(t)-u_{1}(t)-(\eta+\varphi) s_{1}(t) \\
\&(t)=q_{2}(t)-u_{2}(t)+\phi s_{1}(t)-\eta s_{2}(t) \\
w_{2}(t)=-\varepsilon+(\eta+\rho) u_{2}(t) \\
\alpha_{2}^{\&}(t)=\varepsilon+(\eta+\rho) \lambda_{2}(t) \\
\alpha_{3}^{\&}(t)=(\rho+\eta+\varphi) \lambda_{3}(t)-\varphi \lambda_{2}(t)
\end{array}\right.
$$

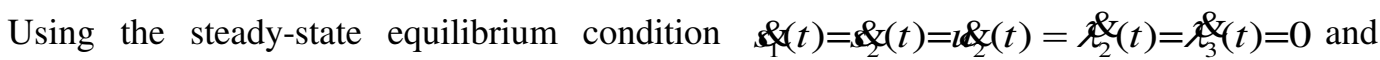
game result and the optimization problem of the manufacturers.

Finishing the proof.

\section{Appendix 2-4}

The proofs for Appendices 1-4 are similar to the proofs for Appendix 1. Therefore, we omit the proofs for them.

\section{Appendix 5}

From Appendix 3 and Appendix 4, we have:

$$
\tau_{1}^{T C}=\tau_{1}^{T N}=\frac{5 \varepsilon}{2(\eta+\phi+\rho)}-\frac{(a-c)}{2} ; \tau_{2}^{T C}=\tau_{2}^{T N}=\frac{(5 \eta+4 \phi) \varepsilon}{4(\eta+\phi)(\eta+\rho)}-\frac{(a-c)}{4}
$$

$$
\tau_{1}^{T N}-\tau_{2}^{T N}=-\frac{(5 \eta+4 \phi) \varepsilon}{4(\eta+\phi)(\eta+\rho)}-\frac{(a-c)(\eta+\phi+\rho)-10 \varepsilon}{4(\eta+\phi+\rho)}<0
$$

Through calculation, we can get $\tau_{1}^{T N}=\tau_{1}^{T C}<\tau_{2}^{T C}=\tau_{2}^{T N}$.

$$
\&(t)=1-\frac{2(1-\delta)}{(\eta+\phi+\rho) \times(1+\delta)+(1-\delta)}=0
$$

$$
\delta^{N C}=\delta^{T C}=\frac{1-(\eta+\phi+\rho)}{1+(\eta+\phi+\rho)}
$$

Finishing the proof.

\section{Appendix 6}


From Appendix 1-4, we can get the emission as follows :

$$
\frac{\partial \pi_{1}}{\partial k_{1}(t)}=k_{1}(t)-\tau_{1}(\mathrm{t})=0 ; \frac{\partial \pi_{2}}{\partial k_{2}(t)}=k_{2}(t)-\tau_{2}(\mathrm{t})=0
$$

696

If there is no tax, manufacturers will not reduce emissions, we have

$$
k_{1}^{N N}=k_{1}^{N C}=k_{2}^{N N}=k_{2}^{N C}=\tau=0
$$

From Appendix 5, we can get $k_{1}^{T N}=k_{1}^{T C}<k_{2}^{T N}=k_{2}^{T C}$.

699 From Appendix 1-4, we have:

$$
u_{1}^{N N}=u_{1}^{T N}=\frac{\varepsilon}{(\eta+\phi+\rho)} ; u_{1}^{N C}=u_{1}^{T C}=\frac{\varepsilon+\varepsilon(\eta+\phi+\rho)}{2(\eta+\phi+\rho)^{2}}
$$

$$
u_{2}=u_{2}^{N C}=u_{2}^{N C}=u_{2}^{T N}=u_{2}^{T C}=\frac{\varepsilon}{(\eta+\rho)}
$$

Through calculation, we can get:

$$
u_{1}^{T C}-u_{1}^{T N}=\frac{\varepsilon-\varepsilon(\eta+\phi+\rho)}{2(\eta+\phi+\rho)^{2}}>0
$$

$$
u_{1}^{T C}-u_{2}^{T C}=\frac{\varepsilon(\eta+\rho)-2 \varepsilon(\eta+\phi+2 \rho)(\eta+\phi+\rho)}{2(\eta+\phi+\rho)^{2}(\eta+\rho)}<0
$$

Therefore, $u_{1}^{T N}=u_{1}^{N N}<u_{1}^{T C}=u_{1}^{N C}<u_{2}$

Finishing the proof.

\section{Appendix 7}

From Appendix 1-4, we can get the upstream pollution stock as follows :

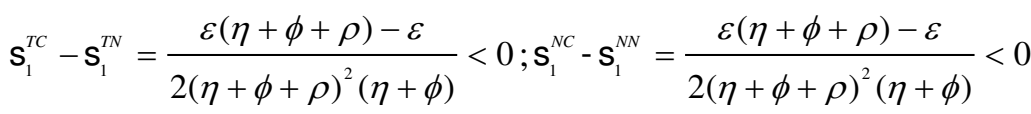

$$
\mathrm{s}_{1}^{N C}-\mathrm{s}_{1}^{T N}=\frac{3(a-c)}{4(\eta+\phi)}+\frac{(5 \eta+4 \phi) \varepsilon}{12(\eta+\phi)^{2}(\eta+\rho)}-\frac{28 \varepsilon(\eta+\phi+\rho)-3 \varepsilon}{6(\eta+\phi+\rho)^{2}(\eta+\phi)}>0
$$

The downstream pollutio stock as follows :

$\mathrm{n}$

$$
\mathbf{s}_{2}^{N C}-\mathbf{s}_{2}^{N N}=\frac{\varepsilon \phi(\eta+\phi+\rho)-\varepsilon \phi}{2 \eta(\eta+\phi+\rho)^{2}(\eta+\phi)}<0 ; \mathbf{s}_{2}^{T C}-\mathbf{s}_{2}^{T N}=\frac{\varepsilon \phi(\eta+\phi+\rho)-\varepsilon \phi}{2 \eta(\eta+\phi+\rho)^{2}(\eta+\phi)}<0
$$




$$
\mathrm{s}_{2}^{N C}-\mathrm{s}_{2}^{T N}=\frac{(a-c)(\eta+4 \phi)}{4 \eta(\eta+\phi)}+\frac{(5 \eta+4 \phi) \varepsilon \phi}{12 \eta(\eta+\phi)^{2}(\eta+\rho)}+\frac{\varepsilon \phi}{2 \eta(\eta+\phi+\rho)^{2}(\eta+\phi)}
$$

$$
-\frac{(25 \eta+20 \phi) \varepsilon}{12 \eta(\eta+\phi)(\eta+\rho)}-\frac{38 \varepsilon \phi-5 \varepsilon(\eta+\phi)}{6 \eta(\eta+\phi+\rho)(\eta+\phi)}>0
$$

$$
\mathrm{s}_{2}^{T N}-\mathrm{s}_{2}^{N N}=\frac{(a-c)(3 \eta+10 \phi)}{12 \eta}+\frac{(5 \eta+4 \phi) \varepsilon \phi}{12 \eta(\eta+\phi)^{2}(\eta+\rho)}
$$

$$
\begin{aligned}
& -\frac{25 \varepsilon \phi-5 \varepsilon(\eta+\phi)}{6 \eta(\eta+\phi+\rho)(\eta+\phi)}-\frac{(25 \eta+20 \phi) \varepsilon}{12 \eta(\eta+\phi)(\eta+\rho)}<0 \\
\mathrm{~s}_{2}^{T C}-\mathrm{s}_{2}^{N C}= & \frac{(a-c)(\eta+4 \phi)}{4 \eta(\eta+\phi)}+\frac{(5 \eta+4 \phi) \varepsilon \phi}{12 \eta(\eta+\phi)^{2}(\eta+\rho)} \\
- & \frac{\varepsilon(25 \eta+20 \phi)}{12 \eta(\eta+\phi)(\eta+\rho)}-\frac{\varepsilon(20 \phi-5 \eta)}{6 \eta(\eta+\phi+\rho)(\eta+\phi)}<0
\end{aligned}
$$

Therefore, $\mathrm{s}_{1}^{T C}<\mathrm{S}_{1}^{N C}<\mathrm{S}_{1}^{T N}<\mathrm{S}_{1}^{N N} ; \mathrm{S}_{2}^{T C}<\mathrm{S}_{2}^{N C}<\mathrm{S}_{2}^{T N}<\mathrm{S}_{2}^{N N}$.

For four policy combinations, we have:

$$
\mathrm{s}_{1}^{N N}-\mathrm{s}_{2}^{N N}=\frac{\varepsilon}{\eta(\eta+\rho)}+\frac{\varepsilon(\phi-\eta)}{\eta(\eta+\phi+\rho)(\eta+\phi)}-\frac{2 \phi(a-c)}{3 \eta(\eta+\phi)}<0
$$

$$
\mathrm{s}_{1}^{N C}-\mathrm{s}_{2}^{N C}=\frac{\varepsilon}{\eta(\eta+\rho)}+\frac{\varepsilon(\phi-\eta)+\varepsilon(\phi-\eta)(\eta+\phi+\rho)}{2 \eta(\eta+\phi+\rho)^{2}(\eta+\phi)}-\frac{2 \phi(a-c)}{3 \eta(\eta+\phi)}<0
$$

$$
\mathrm{s}_{2}^{T N}-\mathrm{s}_{1}^{T N}=\frac{\varepsilon(5 \eta+4 \phi)(\phi-\eta)}{12 \eta(\eta+\phi)^{2}(\eta+\rho)}+\frac{\varepsilon(36 \eta-26 \phi)}{6 \eta(\eta+\phi+\rho)(\eta+\phi)}+\frac{(a-c)(10 \phi-3 \eta)}{6 \eta(\eta+\phi)}-\frac{(37 \eta+32 \phi) \varepsilon}{12 \eta(\eta+\phi)(\eta+\rho)}>0
$$

$$
\mathrm{s}_{2}^{T C}-\mathrm{s}_{1}^{T C}=\frac{(a-c)(13 \phi-6 \eta)}{12 \eta(\eta+\phi)}+\frac{\varepsilon \phi(5 \eta+4 \phi)(1-\eta)}{12 \eta(\eta+\phi)^{2}(\eta+\rho)}-\frac{23 \varepsilon(\phi-\eta)}{6 \eta(\eta+\phi+\rho)(\eta+\phi)}
$$

$$
-\frac{3 \varepsilon(\phi-\eta)}{6 \eta(\eta+\phi+\rho)^{2}(\eta+\phi)}-\frac{\varepsilon(37 \eta+32 \phi)}{12 \eta(\eta+\phi)(\eta+\rho)}>0
$$

Therefore, $\mathrm{s}_{1}^{N N}<\mathrm{S}_{2}^{N N} ; \mathrm{s}_{1}^{N C}<\mathrm{S}_{2}^{N C} ; \mathrm{s}_{1}^{T C}<\mathrm{S}_{2}^{T C} ; \mathrm{s}_{1}^{T C}<\mathrm{S}_{2}^{T C}$.

Finishing the proof.

\section{Appendix 8}

From Appendix 1-4, we can get the production as follows :

$$
q_{1}^{N N}=q_{2}^{N N}=q_{1}^{N C}=q_{2}^{N C}=\frac{a-c}{3}
$$

$$
q_{1}^{T C}=q_{1}^{T N}=\frac{7(a-c)}{12}+\frac{(5 \eta+4 \phi) \varepsilon}{12(\eta+\phi)(\eta+\rho)}-\frac{5 \varepsilon}{3(\eta+\phi+\rho)}
$$




$$
q_{2}^{T C}=q_{2}^{T N}=\frac{a-c}{3}+\frac{5 \varepsilon}{6(\eta+\phi+\rho)}-\frac{(5 \eta+4 \phi) \varepsilon}{6(\eta+\phi)(\eta+\rho)}
$$

$$
q_{1}^{T C}-q_{1}^{N N}=\frac{(a-c)}{4}+\frac{(5 \eta+4 \phi) \varepsilon}{12(\eta+\phi)(\eta+\rho)}-\frac{5 \varepsilon}{3(\eta+\phi+\rho)}>0
$$

$$
q_{2}^{T N}-q_{2}^{N N}=\frac{5 \varepsilon}{6(\eta+\phi+\rho)}-\frac{(5 \eta+4 \phi) \varepsilon}{6(\eta+\phi)(\eta+\rho)}<0
$$

Therefore, $q_{1}^{T C}=q_{1}^{T N}>q_{1}^{N N}=q_{1}^{N C} ; q_{2}^{N N}=q_{2}^{N C}>q_{2}^{T N}=q_{2}^{T C}$.

$$
q_{1}^{T C}-q_{2}^{T C}=q_{1}^{T N}-q_{2}^{T N}=\frac{(a-c)}{4}+\frac{(5 \eta+4 \phi) \varepsilon}{4(\eta+\phi)(\eta+\rho)}-\frac{5 \varepsilon}{2(\eta+\phi+\rho)}>0
$$

Therefore, $q_{1}^{T C}=q_{1}^{T N}>q_{2}^{T C}=q_{2}^{T N} ; q_{1}^{N N}=q_{1}^{N C}=q_{2}^{N N}=q_{2}^{N C}$.

From Appendix 1-4, we can get the production as follows :

736

$$
\begin{aligned}
& W_{1}^{N N}=\frac{(a-c)^{2}}{3}-\varepsilon s_{1}-\frac{u_{1}^{2}}{2}=\frac{(a-c)^{2}}{3}+\frac{\varepsilon^{2}}{(\eta+\phi)(\eta+\rho+\phi)}-\frac{\varepsilon(a-c)}{3(\eta+\phi)}-\frac{\varepsilon^{2}}{2(\eta+\rho+\phi)^{2}} \\
& W_{1}^{N C}=\frac{(a-c)^{2}}{3}-\varepsilon s_{1}-\frac{(1-\delta) u_{1}^{2}}{2}=\frac{(a-c)^{2}}{3}-\frac{\varepsilon(a-c)}{3(\eta+\phi)}+\frac{\varepsilon^{2}(\eta+\rho+\phi)+\varepsilon^{2}}{2(\eta+\phi)(\eta+\rho+\phi)^{2}}
\end{aligned}
$$

$$
\begin{aligned}
& -\frac{(\varepsilon(\eta+\rho+\phi)+\varepsilon)^{2}}{4(\eta+\rho+\phi)^{3}(\eta+\rho+\phi+1)} \\
W_{1}^{T N}= & \frac{2(a-c)^{2}-2(a-c) \tau_{1}-4 \tau_{1}^{2}+\tau_{2}^{2}}{6}-\varepsilon s_{1}-\frac{u_{1}^{2}}{2} \\
= & \frac{(a-c)^{2}}{2}+\frac{1}{6}\left(\frac{\varepsilon(5 \eta+4 \phi)}{4(\eta+\rho)(\eta+\phi)}-\frac{(a-c)}{4}\right)^{2}+\frac{62 \varepsilon^{2}}{12(\eta+\phi)(\eta+\rho+\phi)} \\
= & \frac{5 \varepsilon(a-c)}{6(\eta+\rho+\phi)}-\frac{2}{3}\left(\frac{5 \varepsilon}{2(\eta+\rho+\phi)}-\frac{(a-c)}{2}\right)^{2}-\frac{13 \varepsilon(a-c)}{12(\eta+\phi)}-\frac{\varepsilon^{2}(5 \eta+4 \phi)}{12(\eta+\phi)^{2}(\eta+\rho)}-\frac{\varepsilon^{2}}{2(\eta+\rho+\phi)^{2}} \\
W_{1}^{T C}= & \frac{2(a-c)^{2}-2(a-c) \tau_{1}-4 \tau_{1}^{2}+\tau_{2}^{2}}{6}-\varepsilon s_{1}-\frac{(1-\delta) u_{1}^{2}}{2} \\
= & \frac{(a-c)^{2}}{2}+\frac{28 \varepsilon^{2}(\eta+\rho+\phi)+3 \varepsilon^{2}}{6(\eta+\phi)(\eta+\rho+\phi)^{2}}+\frac{1}{6}\left(\frac{c-a}{4}+\frac{\varepsilon(5 \eta+4 \phi)}{4(\eta+\rho)(\eta+\phi)}\right)^{2}-\frac{2}{3}\left(\frac{c-a}{2}+\frac{5 \varepsilon}{2(\eta+\rho+\phi)}\right)^{2} \\
= & \frac{13(a-c)}{12(\eta+\phi)}-\frac{\varepsilon^{2}(5 \eta+4 \phi)}{12(\eta+\rho)(\eta+\phi)^{2}}-\frac{2 \varepsilon^{2}(\eta+\rho+\phi)+2 \varepsilon^{2}}{8(\eta+\rho+\phi)^{3}}-\frac{5 \varepsilon(a-c)}{6(\eta+\rho+\phi)}
\end{aligned}
$$$$
W_{2}^{N N}=\frac{(a-c)^{2}}{3}-\varepsilon s_{2}-\frac{u_{2}^{2}}{2}=\frac{(a-c)^{2}}{3}+\frac{\varepsilon^{2} \phi}{\eta(\eta+\phi)(\eta+\rho+\phi)}+\frac{\varepsilon^{2}}{\eta(\eta+\rho)}
$$

$$
-\frac{\varepsilon \phi(a-c)}{3 \eta(\eta+\phi)}-\frac{\varepsilon(a-c)}{3 \eta}-\frac{\varepsilon^{2}}{2(\eta+\rho)^{2}}
$$




$$
W_{2}^{N C}=\frac{2(a-c)^{2}}{6}-\varepsilon s_{2}-\frac{u_{2}^{2}}{2}-\frac{\delta u_{1}^{2}}{2} \frac{(a-c)^{2}}{3}+\frac{\varepsilon^{2} \phi}{2 \eta(\eta+\phi)(\eta+\rho+\phi)}+\frac{\varepsilon^{2}}{\eta(\eta+\rho)}
$$

$$
+\frac{\varepsilon^{2} \varphi}{2 \eta(\eta+\phi)(\eta+\rho+\phi)^{2}}-\frac{\varepsilon(a-c)(\eta+2 \phi)}{3 \eta(\eta+\phi)}-\frac{\varepsilon^{2}}{2(\eta+\rho)^{2}}-\frac{\varepsilon^{2}\left(1-(\eta+\rho+\phi)^{2}\right)}{8(\eta+\rho+\phi)^{4}}
$$

$$
W_{2}^{T N}=\frac{2(a-c)^{2}-2(a-c) \tau_{2}+\tau_{1}^{2}-4 \tau_{2}^{2}}{6}-\varepsilon s_{2}-\frac{u_{2}^{2}}{2}
$$

$$
\begin{aligned}
& =\frac{5(a-c)^{2}}{12}+\frac{31 \varepsilon^{2} \phi-5 \varepsilon^{2}(\eta+\phi)}{6 \eta(\eta+\phi)(\eta+\rho+\phi)}+\frac{1}{24}\left(\frac{5 \varepsilon}{\eta+\rho+\phi}-(a-c)\right)^{2}-\frac{1}{24}\left(\frac{\varepsilon(5 \eta+4 \phi)}{(\eta+\rho)(\eta+\phi)}-(a-c)\right)^{2} \\
& -\frac{\varepsilon(a-c)(5 \eta+4 \phi)+\varepsilon(a-c)(7 \eta+20 \phi)(\eta+\rho)-\varepsilon^{2}(37 \eta+32 \phi)}{12 \eta(\eta+\rho)(\eta+\phi)}-\frac{\varepsilon^{2} \phi(5 \eta+4 \phi)}{12 \eta(\eta+\rho)(\eta+\phi)^{2}}-\frac{\varepsilon^{2}}{2(\eta+\rho)^{2}}
\end{aligned}
$$

$$
\begin{aligned}
W_{2}^{T C} & =\frac{2(a-c)^{2}-2(a-c) \tau_{2}+\tau_{1}^{2}-4 \tau_{2}^{2}}{6}-\varepsilon s_{2}-\frac{u_{2}^{2}}{2}-\frac{\delta u_{1}^{2}}{2} \\
& =\frac{5(a-c)^{2}}{12}+\frac{\varepsilon^{2}(37 \eta+32 \phi)}{12 \eta(\eta+\rho)(\eta+\phi)}+\frac{\varepsilon^{2}(23 \phi-5 \eta)(\eta+\rho+\phi)+3 \varepsilon^{2} \phi}{6 \eta(\eta+\phi)(\eta+\rho+\phi)^{2}}+\frac{1}{6}\left(\frac{5 \varepsilon}{2(\eta+\rho+\phi)}-\frac{a-c}{2}\right)^{2}
\end{aligned}
$$

742

$$
\begin{aligned}
& -\frac{2}{3}\left(\frac{\varepsilon(5 \eta+4 \phi)}{4(\eta+\rho)(\eta+\phi)}-\frac{a-c}{4}\right)^{2}-\frac{\varepsilon(a-c)(7 \eta+20 \phi)}{12 \eta(\eta+\phi)}-\frac{\varepsilon^{2} \phi(5 \eta+4 \phi)}{12 \eta(\eta+\rho)(\eta+\phi)^{2}}-\frac{\varepsilon^{2}\left(1-(\eta+\rho+\phi)^{2}\right)}{8(\eta+\rho+\phi)^{4}} \\
& -\frac{\varepsilon(a-c)(5 \eta+4 \phi)}{12(\eta+\rho)(\eta+\phi)}-\frac{\varepsilon^{2}}{2(\eta+\rho)^{2}}
\end{aligned}
$$

$$
W_{1}^{T C}-W_{1}^{T N}=\frac{\varepsilon^{2}(1-(\eta+\rho+\phi))}{2(\eta+\phi)(\eta+\rho+\phi)^{2}}-\frac{\varepsilon^{2}(1-(\eta+\rho+\phi))}{4(\eta+\rho+\phi)^{3}}>0
$$

$$
W_{1}^{N C}-W_{1}^{N N}=\frac{\varepsilon^{2}-\varepsilon^{2} \rho}{2(\eta+\phi)(\eta+\rho+\phi)^{2}}-\frac{\varepsilon^{2}(1+(\eta+\rho+\phi))}{4(\eta+\rho+\phi)^{3}}>0
$$$$
W_{1}^{T N}-W_{1}^{N C}=\frac{(a-c)^{2}}{6}+\frac{1}{6}\left(\frac{\varepsilon(5 \eta+4 \phi)}{4(\eta+\rho)(\eta+\phi)}-\frac{(a-c)}{4}\right)^{2}+\frac{56 \varepsilon^{2}(\eta+\rho+\phi)-6 \varepsilon^{2}}{12(\eta+\phi)(\eta+\rho+\phi)^{2}}+\frac{\varepsilon^{2}(1-(\eta+\rho+\phi))}{4(\eta+\rho+\phi)^{3}}
$$

$$
-\frac{2}{3}\left(\frac{5 \varepsilon}{2(\eta+\rho+\phi)}-\frac{(a-c)}{2}\right)^{2}-\frac{5 \varepsilon(a-c)}{6(\eta+\rho+\phi)}-\frac{3 \varepsilon(a-c)}{4(\eta+\phi)}-\frac{\varepsilon^{2}(5 \eta+4 \phi)}{12(\eta+\phi)^{2}(\eta+\rho)}>0
$$

746

$$
W_{2}^{T C}-W_{2}^{T N}=\frac{3 \varepsilon^{2} \phi}{6 \eta(\eta+\phi)(\eta+\rho+\phi)^{2}}+\frac{1}{8}\left(\frac{5 \varepsilon}{2(\eta+\rho+\phi)}-\frac{a-c}{2}\right)^{2}-\frac{5}{8}\left(\frac{\varepsilon(5 \eta+4 \phi)}{4(\eta+\rho)(\eta+\phi)}-\frac{a-c}{4}\right)^{2}
$$

$$
-\frac{\varepsilon(a-c)(5 \eta+4 \phi)(1-\eta)}{12 \eta(\eta+\rho)(\eta+\phi)}-\frac{\varepsilon^{2}\left(1-(\eta+\rho+\phi)^{2}\right)}{8(\eta+\rho+\phi)^{4}}-\frac{\varepsilon^{2} \phi^{2}}{2 \eta(\eta+\phi)(\eta+\rho+\phi)}<0
$$

$$
W_{2}^{N C}-W_{2}^{N N}=\frac{\varepsilon^{2} \phi(1-(\eta+\rho+\phi))}{2 \eta(\eta+\phi)(\eta+\rho+\phi)^{2}}-\frac{\varepsilon^{2}\left(1-(\eta+\rho+\phi)^{2}\right)}{8(\eta+\rho+\phi)^{4}}>0
$$




$$
W_{2}^{T C}-W_{2}^{N C}=\frac{(a-c)^{2}}{12}+\frac{\varepsilon^{2}(25 \eta+20 \phi)}{12 \eta(\eta+\rho)(\eta+\phi)}+\frac{\varepsilon^{2}(20 \phi-5 \eta)}{6 \eta(\eta+\phi)(\eta+\rho+\phi)}+\frac{1}{6}\left(\frac{5 \varepsilon}{2(\eta+\rho+\phi)}-\frac{a-c}{2}\right)^{2}
$$

$$
-\frac{\varepsilon(a-c)(\eta+4 \phi)}{4 \eta(\eta+\phi)}-\frac{\varepsilon(a-c)(5 \eta+4 \phi)}{12(\eta+\rho)(\eta+\phi)}-\frac{\varepsilon^{2} \phi(5 \eta+4 \phi)}{12 \eta(\eta+\rho)(\eta+\phi)^{2}}-\frac{2}{3}\left(\frac{\varepsilon(5 \eta+4 \phi)}{4(\eta+\rho)(\eta+\phi)}-\frac{a-c}{4}\right)^{2}>0
$$

749

$$
\text { Therefore, } W_{1}^{N N}<W_{1}^{N C}<W_{1}^{T N}<W_{1}^{T C} ; W_{2}^{N N}<W_{2}^{N C}<W_{2}^{T C}<W_{2}^{T N}
$$

750

$$
w^{T C}-w_{2}^{T C}=\frac{1}{6}\left(\frac{\varepsilon(5 \eta+4 \phi)}{4(\eta+\rho)(\eta+\phi)}-\frac{a-c}{4}\right)^{2}+\frac{(a-c)^{2}}{12}+\frac{\varepsilon^{2}}{2(\eta+\rho)^{2}}
$$

751

$$
\begin{aligned}
&-\frac{5}{6}\left(\frac{5 \varepsilon}{2(\eta+\rho+\phi)}-\frac{a-c}{2}\right)^{2}-\frac{\varepsilon^{2}(37 \eta+32 \phi)}{12 \eta(\eta+\rho)(\eta+\phi)}-\frac{23 \varepsilon^{2}(\phi-\eta)(\eta+\rho+\phi)+3 \varepsilon^{2}(\phi-\eta)}{6 \eta(\eta+\phi)(\eta+\rho+\phi)^{2}}>0 \\
& W^{T N}-W^{T N}=\frac{5}{6}\left(\frac{\varepsilon(5 \eta+4 \phi)}{4(\eta+\rho)(\eta+\phi)}-\frac{a-c}{4}\right)^{2}+\frac{1}{12}(a-c)^{2}+\frac{31 \varepsilon^{2}(36 \eta-26 \phi)}{6 \eta(\eta+\phi)(\eta+\rho+\phi)}
\end{aligned}
$$

$$
-\frac{5}{6}\left(\frac{5 \varepsilon}{2(\eta+\rho+\phi)}-\frac{a-c}{2}\right)^{2}-\frac{5 \varepsilon(a-c)}{6(\eta+\rho+\phi)}>0
$$

752

Therefore, $w_{1}^{T C}>w_{2}^{T C} ; w^{T N}>w_{2}^{T N}$

753

Finishing the proof. 